Paediatr Paedolog 2018 · 53 (Suppl 1):S56-S60 https://doi.org/10.1007/s00608-018-0588-z Online publiziert: 21. August 2018

(c) Der/die Autor(en) 2018

CrossMark

\section{Maria Kletečka-Pulker · Sabine Parrag}

Institut für Ethik und Recht in der Medizin, Universitätscampus, Universität Wien, Wien, Österreich

\title{
Videodolmetschen als Kommunikationshilfe bei Flüchtlingen
}

\section{Einwilligung des Patienten als Voraussetzung einer rechtmäßigen Behandlung}

Kommunikation ist ein unverzichtbares Element für die Patientensicherheit sowie für eine qualitätsgesicherte Behandlung und Betreuung. Nicht zuletzt ist es aus juristischer Sicht erforderlich, dass sich die Partner des Behandlungsverhältnisses verständigen können, um die notwendigen Informationen auszutauschen, damit der Patient die erforderliche Einwilligung erteilen kann. Sprachbarrieren können zu Missverständnissen, Fehlern sowie Über- und Unterversorgung von Patienten führen. Darüber hinaus kann es sowohl für die Patienten als auch für die Angehörigen der Gesundheitsberufe sehr belastend sein, wenn sie einander nicht verstehen. Hinzu kommt, dass gerade im Bereich der Pädiatrie die Kommunikation und die Ermittlung des $\mathrm{Pa}$ tientenwillens oft noch komplexer ist, da vor allem bei unmündig-minderjährigen Patienten auch der gesetzliche Vertreter eine wesentliche Rolle spielt.

》) Für die Behandlung nichtdeutschsprachiger Patienten gibt es keine sondergesetzlichen Bestimmungen

Für die Behandlung nichtdeutschsprachiger Patienten gibt es keine sondergesetzlichen Bestimmungen. Daher muss auf allgemeine Grundsätze zurückgegriffen werden, nach welchen für jede rechtmäßige Behandlung eine Einwilligung benötigt wird [12]. Diese wiederum setzt eine entsprechende Aufklärung voraus. Unabhängig von bestimmten Ausnahmefällen, wie drohende Lebensgefahr, ist eine barrierefreie Kommunikation mit dem Patienten essenziell, um diesen über die Diagnose und die geplante Therapie entsprechend aufklären zu können. Wird eine mögliche Behandlung ohne Einwilligung - wenn auch lege artis - durchgeführt, hat der Behandler bzw. der Träger der Krankenanstalt aufgrund der eigenmächtigen Heilbehandlung jegliche Risiken zu verantworten $[8,10]$.

Oftmals fällt es auch Personen mit verhältnismäßig guten Deutschkenntnissen schwer, sich entsprechend zu artikulieren und sich über komplexe medizinische Sachverhalte zu verständigen. Eines der Hauptprobleme in der Praxis liegt für die behandelnden Ärzte in der Beurteilung, ob der Patient die Aufklärung tatsächlich verstanden hat. Dies ist oft schon bei Patienten ohne Sprachbarriere sehr schwierig und zeitintensiv. ${ }^{1}$ Den Arzt trifft grundsätzlich immer eine Kontroll- bzw. Erkundungspflicht, ob bzw. inwieweit ihn der Patient verstanden hat. Im Zweifel muss davon ausgegangen werden, dass der Patient sich nicht ausreichend verständigen kann, und dass daher eine Lösung für die vorhandene Sprachbarriere gefunden werden sollte.

\section{Übernahme der Dolmetsch- kosten}

Anders als in den Bereichen der Justiz oder der Verwaltung gibt es im Gesundheitsbereich keine Regelungen, wer

\footnotetext{
1 Zum Thema Aufklärungsprobleme s. auch zur deutschen Rechtslage [25].
}

für die Kosten eines notwendigen Dolmetschs aufkommen muss. ${ }^{2}$ Allerdings dürfen Krankenanstalten Patienten gemäß $\$ 22$ Abs. 3 und 4 Krankenanstaltenund Kuranstaltengesetz (KAKuG) nicht abweisen, wenn sie unabweisbar oder anstaltsbedürftig sind. Wie aber kann ein Arzt ohne ausreichende Kommunikation feststellen, ob Behandlungsbedürftigkeit vorliegt? So kamen bereits nichtdeutschsprachige Patienten zu Schaden, da sie in der Krankenanstalt nicht behandelt wurden, weil man davon ausgegangen war, dass sie keiner ärztlichen Hilfe bedürften [9]. In diesen Fällen haftete dann die betreffende Krankenanstalt. Aus diesem Grund übernehmen bereits viele Institutionen die Kosten für Video- oder VorOrt-Dolmetsch, da die Kosten eines möglichen Haftungsfalls in der Regel die Kosten für professionelle Dolmetschdienste (z. B. per Video) bei Weitem übersteigen.

\section{Bisherige Lösungsstrategien bei Sprachbarrieren}

Aufgrund der ungeklärten Finanzierungsfrage kommen in der Praxis bisher unterschiedlichste Notlösungen zum Einsatz, wobei manche davon besonders kritisch $\mathrm{zu}$ hinterfragen sind. Im Vorfeld des Pilotprojekts „Videodolmetschen im Gesundheitswesen " führten das Institut für Ethik und Recht in der Medizin und die Österreichische Plattform Patientensicherheit eine Erhebung durch, um sichtbar zu machen, wie bisher mit sprachlichen Barrieren umgegangen wurde $[6,7,13]$. Eben-

\footnotetext{
2 Zur Frage der Finanzierung von Dolmetschen s. ausführlich [11].
} 
so häufig wie Angehörige der Patienten wurden fremd- bzw. mehrsprachige Mitarbeiter, die beim niedergelassenen Arzt oder in der Krankenanstalt beschäftigt waren, als Sprachmittler herangezogen. Oftmals gibt es eigene Listen mit den infrage kommenden Mitarbeitern, wobei diese keine Qualifikation als Dolmetsch aufweisen und daher auch nur laienhaft dolmetschen können. Die Studie ergab, dass bisher nur sehr selten professionelle Dolmetsche bei Kommunikationsproblemen zugezogen wurden.

\section{》) Die Inanspruchnahme von Kindern und Jugendlichen als Sprachmittler ist problematisch}

Das ist höchst problematisch (s. dazu Untersuchungen von Flores et al. [4], Pöchhacker [19] oder Sator [22]). Besonders kritisch zu hinterfragen ist die Inanspruchnahme von Kindern und Jugendlichen als Sprachmittler, da diese Lösungsstrategie aus rechtlicher und ethischer Perspektive problematisch ist. Gründe sind das hohe Fehlerrisiko und die teils unzumutbaren Belastungen für die Kinder und Jugendlichen. Verantwortung und die Bringschuld zur gelingenden Kommunikation haben in der Praxis de facto die Patienten, sodass sie selbst oft Sprachmittler mitnehmen [17].

Einen vorhandenen Handlungsbedarf und notwendige Veränderung der gängigen Praktiken verdeutlichen auch die Ergebnisse der Fragebogenerhebung des Pilotprojekts. Die Mehrheit aller befragten Mitarbeiter gab an, mit der derzeit vorherrschenden Praxis zur Überwindung von Sprachbarrieren wenig bis gar nicht zufrieden zu sein [13].

Oftmals fehlt es bereits in der Führungsebene am Problembewusstsein für den tatsächlichen Bedarf an institutionell zur Verfügung gestellten professionellen Lösungsstrategien. Hinzu kommt auch auf Seiten der Angehörigen der Gesundheitsberufe ein fehlendes Problembewusstsein bezüglich der Notwendigkeit professioneller Dolmetsche, was wiederum mit fehlendem Wissen über die erforderliche berufliche Quali- fikation zur Ausübung dieser Tätigkeit zusammenhängt. $^{3}$

\section{Kinder und Jugendliche als Laiendolmetsche}

Dass Kinder und Jugendliche nichtdeutschsprachiger Eltern einen Großteil der benötigten Dolmetschleistungen abdecken, ist nicht nur in Österreich eine gängige und langjährige Praxis [14, 24]. ${ }^{4}$ Die Erhebung im Rahmen des Pilotprojekts „Videodolmetschen im Gesundheitswesen“ ergab, dass $81 \%$ der befragten Angehörigen der Gesundheitsberufe Kinder als Sprachmittler heranziehen.

Eine von Ebden et al. [3] durchgeführte Untersuchung über Dolmetschleistungen von Kindern und anderen Familienangehörigen ergab, dass jedenfalls $16 \%$ der als einfach eingestuften Fragen falsch oder auch gar nicht gedolmetscht wurden und dass dies bei komplexeren Fragen sogar $82 \%$ betraf. Dabei wurden besonders häufig anatomische Begriffe ungenau oder Symptome falsch wiedergegeben. Nicht zuletzt gab es große Schwierigkeiten beim Übersetzen von Fachbegriffen [3].

\section{》) Die Dolmetschleistungen von Kindern und anderen Familienangehörigen sind stark fehlerhaft}

Im Jahr 2009 wurde auch in Deutschland eine Befragung von Kinder- und Jugendärzten durchgeführt, die aufzeigte, dass Kinder und Jugendliche bei An-

3 Auf Basis des Pilotprojekts "Videodolmetschen im Gesundheitswesen" konnte die Dienstleistung Videodolmetschen bereits in Österreich und Deutschland erfolgreich etabliert werden, wobei die Qualifizierung als Dolmetsch ein zentrales Qualitätskriterium ist, s. [23].

${ }^{4}$ Ein guter Überblick hinsichtlich des internationalen Vorhandenseins und der Allgegenwärtigkeit von Kindern und Jugendlichen als Sprachmittler findet sich z. B. in der Sonderausgabe der Zeitschrift MediAzioni Nr. 10 aus dem Jahr 2010 mit dem Schwerpunkt "Child Language Brokering: Trends and Patterns in Current Research" [1]. gehörigen der Gesundheitsberufe eine große Akzeptanz als Sprachmittler erreichen. Insbesondere von Ärzten im niedergelassenen Bereich wurde die Einbeziehung von Kindern und Jugendlichen als Sprachmittler mangels Alternativen als sehr sinnvoll erachtet [15].

\section{Professionelle Dolmetsche}

Eines der Hauptprobleme in diesem Zusammenhang ist - neben der Frage der Finanzierung - die Annahme, dass eine grundsätzliche Sprachkompetenz, wie sie viele Laiendolmetsche haben, für das Dolmetschen ausreicht. Für professionelles Dolmetschen bedarf es allerdings translatorischer Kompetenz, reine Sprachkompetenz ist nicht ausreichend [13, 18, 20].

Beachtet werden muss, dass mehrsprachige Kinder und Jugendliche ihre sogenannte „angeborene“ Dolmetschfähigkeit - wenn überhaupt - nur dann effizient einsetzen können, wenn der Kontext innerhalb ihres Sprach- und Erfahrungshorizonts liegt [21]. Dabei kommt es gerade im medizinischen Bereich, wie beispielsweise auch bei Behörden oder in der Schule, etwa in ElternLehrer-Gesprächen, besonders leicht zur Überschreitung des entsprechenden kindlichen Horizonts. In sogenannten „geschützten“ Kontexten hingegen, wie etwa bei Gesprächen zu Hause mit Freunden oder Bekannten oder beim Einkaufen, kann die Tätigkeit sehr wohl auch positive Auswirkungen auf die Entwicklung der Kinder haben.

Zum Thema Kinder als Dolmetsche ist vor allem kritisch zu hinterfragen, wessen Verantwortung und Entscheidung es letztendlich sein kann bzw. soll, Kinder als Laiendolmetsche in heiklen Situationen heranzuziehen. Soll oder darf es wirklich allein die Entscheidung der Eltern sein oder ist es erforderlich, auch als Institution und somit als Angehörige der Gesundheitsberufe hier Position zu beziehen, um Kindern oder Jugendlichen diese Verantwortung nicht zumuten zu müssen und diese zu schützen?

Ein wesentlicher Schritt zur langfristigen und nachhaltigen Veränderung der unbefriedigenden aktuellen Lage wäre jedenfalls die Institutionalisierung profes- 
sioneller Lösungsstrategien und das Festlegen von Leitlinien zur Überwindung von Sprach- und Kommunikationsbarrieren im Gesundheits- und Sozialbereich. Dies wird von Experten schon lange vehement gefordert. Voraussichtlich braucht es aber eine gesetzliche Klarstellung, damit von der gängigen Praxis abgegangen wird, Kinder und Jugendliche in oft heiklen Situationen als Sprachmittler heranzuziehen. In vielen Bereichen der Justiz und Verwaltung wäre eine solche Vorgangsweise aufgrund der Gesetzeslage rechtswidrig. Bis zu einer möglichen gesetzlichen Regelung bleibt es in der Verantwortung der einzelnen Institution bzw. der Angehörigen eines Gesundheitsberufs, im Sinne der Mitarbeiterwie auch der Patientensicherheit professionelle Lösungen $\mathrm{zu}$ finden, wie etwa den Einsatz von Präsenz- oder Videodolmetschen.

\section{Videodolmetschen - ein neues Tool für das Gesundheitswesen}

Der Einsatz von Videokonferenzsystemen stellt international längst keine Neuheit mehr dar, dies schließt natürlich ebenso die Nutzung dieser innovativen Technologien im medizinischen Setting ein. Durch den modernen Stand der heutigen Kommunikationstechnologie und ihre bereits weit fortgeschrittene Integration in das tägliche Leben ist Videokommunikation für einen großen Teil der Bevölkerung schon fast alltäglich geworden. Den sich dadurch eröffnenden neuen Möglichkeiten zur flexibleren und vernetzteren Kommunikation über Landesgrenzen hinweg sind damit nahezu keine Grenzen mehr gesetzt.

Während Videodolmetschen im Gesundheitsbereich zur Überwindung von Sprachbarrieren vereinzelt - beispielsweise in den USA oder in Schweden längst fixer Bestandteil der Versorgung fremdsprachiger Patienten ist, wurde im deutschsprachigen Raum erst vor wenigen Jahren mit der Etablierung eines professionellen Videodolmetschservice begonnen, initiiert durch das Pilotprojekt [13] der Österreichischen Plattform Patientensicherheit und des Instituts für

Paediatr Paedolog 2018 · 53 (Suppl 1):S56-S60 https://doi.org/10.1007/s00608-018-0588-z (c) Der/die Autor(en) 2018

\section{Kletečka-Pulker · S. Parrag}

\section{Videodolmetschen als Kommunikationshilfe bei Flüchtlingen}

\section{Zusammenfassung}

Sprachbarrieren mit nichtdeutschsprachigen Patienten sind für Angehörige von Gesundheitsberufen sowie Krankenhausträger nicht nur aus medizinischer Sicht eine große Herausforderung, sondern stellen auch ein großes Haftungsrisiko dar. Neben der im Regelfall ungeeigneten Verwendung von Laiendolmetschen und dem Einsatz von oftmals nur schwer verfügbaren und finanzierbaren professionellen Dolmetschen vor Ort ist der Einsatz eines Videodolmetschsystems eine sehr gute Lösung. Das System ist unkompliziert und löst Übersetzungsprobleme vor Ort. Hunderte professionelle Dolmetsche stehen rund um die Uhr zur Verfügung. Innerhalb von 2 min können diese auf Knopfdruck - unter Einhaltung der strengen datenschutzrechtlichen Vorgaben - zugeschaltet werden. Mittlerweile gehört der Einsatz von Videodolmetschen in vielen Spitälern in Österreich und Deutschland zum
Alltag und ist ein Qualitätskriterium für eine Behandlung oder Betreuung von Patienten. Anders ist die Situation im niedergelassenen Bereich, da hier die Finanzierung noch nicht geklärt ist. Aus diesem Grund ist es für nichtdeutschsprachige Patienten oftmals schwer, Ärzte im niedergelassenen Bereich zu finden, die trotz Sprachbarriere die Betreuung und Behandlung übernehmen. Eine klare Regelung zur professionellen Überwindung von Sprachbarrieren wäre daher sehr begrüßenswert und würde sowohl die rechtliche Situation entschärfen als auch zu einer Entlastung der Krankenanstalten führen.

Schlüsselwörter

Kommunikationsprobleme · Aufklärung · Rechtssicherheit · Patientensicherheit . Minderjährige

\section{Video interpreters as communcation aids for refugees}

\section{Abstract}

Language barriers with non-German speaking patients are not only a great challenge for healthcare professionals and hospital managment from a medical perspective but also represent a huge liability risk. In addition to the as a rule inappropriate use of lay interpreters and the employment of professional interpreters who often have limited availability and are expensive, the use of a video interpreter system is a very good alternative solution. The system is uncomplicated and resolves translation problems on site. Hundreds of professional interpreters are available around the clock. Within 2 min these can be reached at the press of a button, in compliance with the strict prerequisites for data protection. The use of video interpreters is nowadays routine in many hospitals in Austria and Germany and is a quality criterion for treatment and care of patients. The situation in private practices is different because the financing has not yet been clarified. For this reason it is often difficult for non-German speaking patients to find physicians in private clinics, willing to handle medical care, regardless of the language barrier. A clear regulation for professionally surmounting the language barrier would therefore be desirable and would de-escalate the legal situation and also lead to a relief of the healthcare institutions.

\section{Keywords}

Communication barriers - Informed consent . Legal certainty · Patient safety · Minors 
Ethik und Recht in der Medizin der Universität Wien.

Der Einsatz von remote ${ }^{5}$ zugeschalteten, geschulten Gesundheitsdolmetschen bietet eine neue qualitätsgesicherte Alternative zu den bisher praktizierten (vorwiegend nicht qualitätsgesicherten) Lösungsstrategien zur Überwindung von Sprachbarrieren im österreichischen Gesundheitswesen, aber auch zum Modell des Telefondolmetschens [16], das insbesondere in der Schweiz weitverbreitet ist. Die Ergebnisse des Pilotprojekts zeigten klar, dass das Tool, Videodolmetschen, eine verlässliche Möglichkeit bietet, potenzielle Schäden bei Patienten und unnötigen Mehraufwand und Belastung durch Sprachbarrieren für Angehörige der Gesundheitsberufe wesentlich zu minimieren. Unbestritten bietet das Hinzuziehen professionell ausgebildeter Gesundheitsdolmetsche ein zusätzliches Sicherheitsnetz zur Vorbeugung von Vorfällen aufgrund sprachlicher und kulturspezifischer Kommunikationsbarrieren, wie dies bereits durch eine Reihe von Studien belegt werden konnte [5, 19, 22].

Ein vielfach von den Angehörigen der Gesundheitsberufe betonter Aspekt war jener der potenziellen Arbeitserleichterung durch den Einsatz von Videodolmetschen. Dabei wurde besonders die empfundene Notwendigkeit einer schnellen Verfügbarkeit von Lösungsstrategien hervorgehoben, und dass Videodolmetschen dieser Anforderung jedenfalls gerecht wurde.

In einer aktuell vom Institut für Ethik und Recht in der Medizin durchgeführten Follow-up-Studie soll nun erhoben werden, inwieweit sich Videodolmetschen im Gesundheitswesen als fixer Bestandteil einer qualitätsgesicherten Gesundheitsversorgung nichtdeutschsprachiger Patienten etablieren konnte, und inwiefern eine Veränderung des Nutzungsverhaltens, insbesondere auch in Bezug auf das Heranziehen von Kin-

\footnotetext{
5 Der englische Begriff des "remote interpreting" beschreibt den Modus des physischen Getrenntseins des Dolmetschs von den anderen in der Dolmetschung involvierten Personen [2]. Dies trifft aufFormen des Dolmetschens wiejene des Telefon-oder Videodolmetschens zu.
}

dern und Jugendlichen als Sprachmittler, im Vergleich zu den Ergebnissen der Pilotstudie erkennbar ist.

\section{Fazit}

- Jede rechtmäßige Behandlung erfordert eine Einwilligung nach entsprechender Aufklärung. Für die Behandlung nichtdeutschsprachiger Patienten gibt es keine sondergesetzlichen Bestimmungen.

- Den Arzt trifft grundsätzlich immer eine Kontroll- bzw. Erkundungspflicht, ob bzw. inwieweit inn der Patient verstanden hat. Im Zweifel muss eine Lösung für die vorhandene Sprachbarriere gefunden werden.

- Die Übernahme der Dolmetschkosten ist im Gesundheitsbereich nicht geregelt.

- Bislang werden nur sehr selten professionelle Dolmetsche bei Kommunikationsproblemen hinzugezogen.

- Die Inanspruchnahme von Kindern und Jugendlichen als Sprachmittler ist aus rechtlicher und ethischer Perspektive problematisch. Gründe sind das hohe Fehlerrisiko und die teils unzumutbaren Belastungen für die Kinder und Jugendlichen.

- Voraussichtlich braucht es eine gesetzliche Klarstellung, damit von der gängigen Praxis abgegangen wird, Kinder und Jugendliche in oft heiklen Situationen als Sprachmittler heranzuziehen.

- Neue, moderne Lösungsstrategien zur Überwindung von Sprachbarrieren, wie bspw. Telefon- oder Videodolmetschen bieten mittlerweile eine vielversprechend kosteneffiziente und flexible, aber vor allem rechtssichere und ethisch vertretbare Alternative zum Einsatz von Laiendolmetschen.

\section{Korrespondenzadresse}

\begin{tabular}{|c|c|}
\hline $\begin{array}{l}\text { O DAS PORTRAIT - } \\
\text { Studio für Photo- } \\
\text { graphie }\end{array}$ & $\begin{array}{l}\text { Dr. M. Kletečka-Pulker } \\
\text { Institut für Ethik und } \\
\text { Recht in der Medizin, } \\
\text { Universitätscampus, } \\
\text { Universität Wien } \\
\text { Spitalgasse 2-4, Hof 2.8, } \\
\text { 1090 Wien, Österreich } \\
\text { maria.kletecka-pulker@ } \\
\text { univie.ac.at }\end{array}$ \\
\hline $\begin{array}{l}\text { (0) Fotoatelier } \\
\text { Andrea Bichl }\end{array}$ & $\begin{array}{l}\text { Mag. S. Parrag } \\
\text { Institut für Ethik und } \\
\text { Recht in der Medizin, } \\
\text { Universitätscampus, } \\
\text { Universität Wien } \\
\text { Spitalgasse 2-4, Hof 2.8, } \\
1090 \text { Wien, Österreich }\end{array}$ \\
\hline
\end{tabular}

Dr. Maria Kletečka-Pulker ist Juristin und Geschäftsführerin der Österreichischen Plattform Patientensicherheit sowie des Instituts für Ethik und Recht in der Medizin der Universität Wien. Zudem ist sie Mitglied der Bioethikkommission beim Bundeskanzleramt.

Mag. Sabine Parrag ist Kultur- und Sozialanthropologin und seit 2009 wissenschaftliche Mitarbeiterin am Institut für Ethik und Recht in der Medizin der Universität Wien und der Österreichischen Plattform Patientensicherheit.

Funding. Open access funding provided by University of Vienna.

\section{Einhaltung ethischer Richtlinien}

Interessenkonflikt. M. Kletečka-Pulker und S. Parrag geben an, dass kein Interessenkonflikt besteht.

Dieser Beitrag beinhaltet keine von den Autoren durchgeführten Studien an Menschen oder Tieren.

Open Access. Dieser Artikel wird unter der Creative Commons Namensnennung 4.0 International Lizenz (http://creativecommons.org/licenses/by/4.0/deed. de) veröffentlicht, welche die Nutzung, Vervielfältigung, Bearbeitung, Verbreitung und Wiedergabe in jeglichem Medium und Format erlaubt, sofern Sie den/die ursprünglichen Autor(en) und die Quelle ordnungsgemäßnennen, einen Link zur Creative Commons Lizenz beifügen und angeben, ob Änderungen vorgenommen wurden.

\section{Literatur}

1. Antonini R (2010) Child language brokering: trends and patterns in current research. Zeitschrift MediAzioni Nr. 10 (Sonderausgabe)

2. Braun S (2013) Keep your distance? Remote interpreting in legal proceedings: A critical assessment of a growing practice. Interpreting 15(2):200-228

3. Ebden, Bhatt, Carey, Harrison (1988) The bilingual consultation. Lancet 331:347 


\section{Leitthema}

4. Flores et al (2003) Errors in medical interpretation and their potential clinical consequences in pediatric encounters. Pediatrics. https://doi.org/ 10.1542/peds.111.1.6

5. Flores $G$ (2005) The impact of medical interpreter services on the quality of health care: A systematic review. Med Care Res Rev 62:255-299

6. IERM, ANetPAS (2013) Teil-Projekt „Sprachbarriere - Eine Befragung von Wiener Kinderärzten zu Lösungsstrategien bei der Versorgung nichtdeutschsprachiger Patienten im niedergelassenen Bereich"

7. IERM, ANetPAS (2015) Teil-Projekt „Gesundheitsförderung via Videodolmetschen"im niedergelassenen Bereich des Projektes "Qualitätssicherung in der Versorgung nicht-deutschsprachiger Patienten-Videodolmetschen im Gesundheitswesen"

8. Kletečka (2013) Kap II.1.2.3. In: Aigner, Kletečka, Kletečka-Pulker, Memmer (Hrsg) Medizinrecht

9. Kletečka (2010) Eigenverschulden des Patienten an der Behandlungsbedürftigkeit und Arzthaftung. Eine weitere Anmerkung zur Entscheidung $4 \mathrm{Ob}$ 36/10p. Zivilr Aktuell 14:276

10. Kletečka, Neumayer (2012) Die Grenzen der ärztlichen Aufklärungspflicht insbesondere bei Schulimpfaktionen. RMed 2:46

11. Kletečka-Pulker (2013) Patientenrecht auf muttersprachliche Aufklärung? Videodolmetschen neue Wege der Kommunikation mit MigrantInnen im Gesundheitsbereich.In:Kaelin, Kletečka-Pulker Körtner (Hrsg) Wieviel Deutsch braucht man, um gesund zu sein?, S55

12. Kletečka-Pulker (2016) Sprachbarrieren im Gesundheitsbereich - Reduktion des Haftungsrisikos durch Videodolmetschen. GesR 4:206

13. Kletečka-Pulker, Parrag (2015) Endbericht Videodolmetschen

14. Kletečka-Pulker, Parrag (2016) Kinder als Laiendolmetscher im Gesundheits- und Sozialbereich. Wenn Kinder dolmetschen: Haftungsrisiken und Probleme einer gängigen Praxis. Interdiszip Z Familienr 2:101

15. Langer, Schaper, Gupta, Porst, Ostermann (2013) Sprachbarrieren in der Betreuung von Patienten mit Migrationshintergrund - Ergebnisse einer Pilotstudie zu Erfahrungen von Kinder- und Jugendärzten. Klin Padiatr 225:96

16. http://0842-442-442.ch/der-nationaletelefondolmetschdienst.html. Zugegriffen: 20.07.2018

17. Papic, Malak, Rosenberg (2012) Patient education and counselling, S205

18. Pöchhacker (2000) Dolmetschen. Konzeptuelle Grundlagen und deskriptive Untersuchungen, $\mathrm{S} 42$

19. Pöchhacker F (2008) Krankheit, Kultur, Kinder, Kommunikation: Die Nichte als Dolmetscherin. Curare 31(2/3):133-142

20. Pöllabauer (2005) I don't understand your English, Miss. In:Dolmetschen bei Asylanhörungen, S59

21. Rajič (2006) Ich habe mich ganz peinlich gefühlt. In: Grbić, Pöllabauer (Hrsg) Forschungen zum Kommunaldolmetschen in Österreich: Problemstellungen, Perspektiven und Potentiale, S153

22. Sator M (2013) Familiendolmetschung vs. Professionelle Dolmetschung - eine Fallstudie. In Menz F (Hrsg) Migration und medizinische Kommunikation, S33-146

23. SAVD Videodolmetschen $\mathrm{GmbH}$ (2016) Videodolmetschen. Einfach. Persönlich. www. videodolmetschen.com.Zugegriffen: 04.04.2016

24. Schmidt-Glenewinkel (2013) Kinder als Dolmetscher, $\mathrm{S} 55$
25. Spickhoff (2010) Spezielle Patientenrechte für Migranten? Juristische und rechtsethische Überlegungen. In: Deutscher Ethikrat (Hrsg) Migration und Gesundheit. Kulturelle Vielfalt als Herausforderung für die medizinische Versorgung, S65 